\title{
Novel indices of ventricular repolarization to screen post myocardial infarction patients
}

\author{
Carlos Alvarado-Serrano ${ }^{\mathrm{a}, *}$, Juan Ramos-Castro $^{\mathrm{b}}$, Ramon Pallàs-Areny ${ }^{\mathrm{b}}$ \\ a Department of Electrical Engineering, Research Center and Advanced Studies of the National Polytechnic Institute \\ (CINVESTAV), México D.F., México \\ ${ }^{\mathrm{b}}$ Department of Electronic Engineering, Technical University of Catalonia (UPC), Barcelona, Spain
}

Received 25 October 2004; accepted 11 March 2005

\begin{abstract}
We propose novel indices of ventricular repolarization intervals, the JTp/JT, Tpe/JTp and Tpe/JT ratios. These indices have been compared with the duration of the ventricular repolarization intervals and other ratios in 17 normal subjects and 17 patients with old myocardial infarction. In the intervals and other ratios, the best separation between groups is obtained with the Tpe/QTp and Tpe/QT ratios with 94\% sensitivity and 82\% specificity, the proposed ratios increased sensitivity to $100 \%$ and specificity to $94 \%$. These indices should be further tested to determine their usefulness in discriminating between OMI patients with and without susceptibility to ventricular arrhythmias.

(c) 2005 Elsevier Ltd. All rights reserved.
\end{abstract}

Keywords: Ventricular repolarization; QT interval; JT interval; JTp interval; Tpe interval; Intervals ratios; Myocardial infarction

\section{Introduction}

Prolongation of the QT interval is associated with a high risk of ventricular arrhythmias and sudden cardiac death in post myocardial-infarction (post-MI) patients. However, this index has a low sensitivity and specificity due in part to: (a) an inaccurate measurement of the QT interval because of different definitions for the end of the $T$ wave, (b) the measurement leads used and (c) the influence of the heart rate $(\mathrm{HR})$ and the autonomic tone [1,2].

\footnotetext{
* Corresponding author. Tel.: +52 55 5061 3800x6216; fax: +52 5550613981.

E-mail address: calvarad@mail.cinvestav.mx (C. Alvarado-Serrano).
} 
Other intervals of ventricular activity have been considered to assess the risk of malign ventricular arrhythmias in post-MI patients. The JT interval better describes the duration of the repolarization than the QT interval when there is a prolongation of the QRS interval in patients [3]. In another study, the JT interval was significantly longer in post-MI patients with delayed ventricular conduction and susceptibility to sustained ventricular tachycardia (VT) than in controls [4]. On the other hand, a QRS prolongation can contribute to the risk of cardiac death independently of repolarization abnormalities $[5,6]$.

The QTpeak (QTp) interval comprises more than 50\% of the ventricular repolarization and it is easier to measure than the QT interval. However, its suitability as a measure of the repolarization instead of the QT interval remains unclear because it is known that the Tpeak-Tend (Tpe) interval is influenced by disease and exercise, yet its variation is not reflected in that of the QTp interval [7]. Although it has been reported that the QTp and Tpe intervals are longer in post-MI patients than in normal subjects, those differences were only significant for the QTp interval [8]. A different study in patients with coronary artery disease susceptible to ventricular fibrillation (VF) also showed that the QTp and JTpeak (JTp) intervals were significantly longer [6].

The Tpe interval corresponds to the final part of the ventricular repolarization and it has been proposed as a more representative measure of that repolarization, because it is less dependent on HR, autonomic modulations and QRS duration than the QT and QTp intervals [9]. Furthermore, it has been experimentally shown that it correlates with the dispersion of the repolarization [10], and it has been hypothesized that it represents the transmural dispersion of the repolarization, defined as the difference in action potential duration between mid-myocardial M-cells, epicardial and endocardial cells [11].

In agreement with this last hypothesis, a separate study has shown that the Tpe interval and the Tpe/QT ratio are significantly larger in post-MI patients with VT than in patients without VT [12]. In other studies, however, the Tpe/QT and Tpe/QTp ratios did not display any significant difference in patients with coronary disease compared with normal subjects $[13,14]$. Another variable that can simplify the measurement of the dispersion of the repolarization is the QTp/QT ratio in the V3 lead, because it has been demonstrated that it has an acceptable correlation with the dispersion of the ventricular repolarization invasively evaluated in the human heart [15]. But its clinical value in post-MI patients has not been assessed to date.

In summary, clinical studies with different intervals of ventricular activity have shown their inconsistency and low sensitivity and specificity for diagnosis and/or prognosis. The objective of this work is to evaluate novel indices based in ratios between intervals of ventricular repolarization in normal subjects and in patients with old myocardium infarct (OMI).

\section{Methods}

\subsection{Subjects and data acquisition}

The study group has been 17 normal subjects (13 men, 4 women; age $40 \pm 12$ years) and 17 OMI patients ( 13 men, 4 women; age $44 \pm 15$ years). Of the 34 records analyzed, 28 records belonged to the two 15-lead CSE (Common Standards for Quantitative Electrocardiography) data bases (12-lead ECG plus Frank's orthogonal XYZ leads) [16]. These databases are available for testing and development of ECG wave recognition and measurement programs, and consist of a set of ECGs with different morphologies, normal as well as pathological (OMI), sampled at $500 \mathrm{~Hz}$. 
Fourteen of those records (EO1 set files: 006, 009, 023, 024, 025, 026, 066, 118 and 124; EO2 set files: 020, 073, 080, 116 and 119) were from the 3-lead data base in which 3 leads had been recorded simultaneously. The other 14 records (MO1 set files: 088, 091, 097, 099, 101, 114, 124 and 125; MO2 set files: 088, 098, 104, 105, 111 and 096) were from the multilead data base in which 15 leads had been simultaneously recorded.

The remaining 6 records were obtained from normal subjects at rest where 12 simultaneous derivations were amplified (programmable gain from 500 to 2500 , bandwidth $0.025-500 \mathrm{~Hz}$ ) by a proprietary highresolution electrocardiography system [17]. The ECG signals were sampled for $5 \mathrm{~min}$ at $1000 \mathrm{samples} / \mathrm{s}$ by a 12 bit, plug-in PC card (DT21EZ, Data Translation) controlled by Asyst ${ }^{\circledR}$ (Asyst Software Technologies, Inc.). Afterwards these records were resampled at 500 samples/s.

In the pathological records with OMI (EO1 set files: 006, 009, 023, 024, 025, 026, 066, 118 and 124; EO2 set files: 020, 073, 080, 116 and 119; MO1 set files: 091 and 114; MO2 set file: 096) the infarct was located by means of the ECG for all the patients. There were 11 inferior infarcts and 6 anterior infarcts. The criteria used to diagnose an OMI were a pathological $Q$ wave longer than 40 ms or a minimal amplitude larger than a fourth of that of the QRS complex and an isoelectric ST segment [18]. All records had a normal sinusal rhythm and none had a complete His bundle branch block.

\subsection{Data processing and analysis}

The computer algorithms to detect the points of interest in the QRS complex and $T$ wave, necessary to measure the different time intervals, have been developed by ourselves (written in Matlab ${ }^{\circledR}$, The MathWorks Inc.) and were based on the wavelet transform (WT) [19]. The validation process of the three designed algorithms has been performed by using 25 records of the well-annotated reference database for ECG measurement, the CSE multi-lead measurement database [16], in which the gold standard had been derived by an international group of cardiologists who visually determined the on- and -offset points of $P, \mathrm{QRS}$ and $T$ waves [20]. In those 15-lead records, the earliest onset ( $Q i$ ) of the QRS complex and the latest offsets ( $\mathrm{Se}$ and $\mathrm{Te}$ ) of the QRS complex and $T$ wave of each record were detected, and the measurements were within the tolerance limits for deviations with respect of the manual measurements determined by the CSE experts [19,21].

The following intervals have been measured for each subject: QT (from $Q$ wave onset to $T$ wave end, defined as the return to the isoelectric line), QTp (from $Q$ wave onset to $T$ wave apex), QRS (from $Q$ wave onset to $S$ wave end), JT (from $S$ wave end to $T$ wave end), JTp (from $S$ wave end to $T$ wave apex) and Tpe (from $T$ wave apex to $T$ wave end). The duration of the intervals has been measured from the V3 lead for healthy subjects [22], the V2 or V6 leads for patients with anterior infarct, and D2 or aVF leads for patients with inferior infarct [23]. The duration of all the intervals of ventricular activity and the preceding RR intervals have been determined from the average of three consecutive beats. The QT, QTp, JT, and JTp intervals have been corrected according to Bazett's formula, but the Tpe and QRS intervals and the ratios QTp/QT, Tpe/QTp, Tpe/QT, JTp/JT, Tpe/JTp, and Tpe/JT have not been corrected.

The intervals corrected according to Bazett's formula (QTc, QTpc, JTc, and JTpc), the uncorrected intervals (Tpe and QRS) and the intervals ratios (QTp/QT, Tpe/QTp, Tpe/QT, JTp/JT, Tpe/JTp, and Tpe/JT) corresponding to normal subjects and OMI patients, have been compared by the Wilcoxon rank sum test. Two-tailed $p<0.05$ has been considered statistically significant. 


\section{Results}

Table 1 shows the mean $(m)$, standard deviation (sd) and level of significance $(p)$ of the durations of the ventricular activity intervals corrected according to Bazett's formula except QRS and Tpe intervals, and the HR in the study groups. The mean HR was $(71 \pm 8)$ beats/min in normal subjects and $(69 \pm 8)$ beats/min in OMI patients. This difference is not significant and therefore the HR influence in both groups is similar. There was no significant difference between the QRS interval in both groups either, since in normal subjects the duration was $(95 \pm 10) \mathrm{ms}$, and in OMI patients $(94 \pm 10) \mathrm{ms}$.

The QTc, QTpc, JTc, and JTpc intervals are longer in OMI patients than in normal subjects. These differences are significant for the intervals QTpc [ $(320 \pm 19) \mathrm{ms}$ vs. $\left.(290 \pm 20) \mathrm{ms}, p=6 \times 10^{-4}\right]$, and JTpc $\left[(220 \pm 17) \mathrm{ms}\right.$ vs. $\left.(187 \pm 14) \mathrm{ms}, p=1.9 \times 10^{-5}\right]$ (Fig. 1$)$.

The Tpe interval is significantly shorter in OMI patients than in normal subjects $[(96 \pm 13) \mathrm{ms}$ vs. $(114 \pm 8) \mathrm{ms}, p=1.7 \times 10^{-4}$ ] (Fig. 1).

Table 2 shows the mean $(m)$, standard deviation (sd), level of significance $(p)$, cutoff values, sensitivity and specificity of intervals (QTpc, JTpc and Tpe) and intervals ratios in the study groups. In the intervals, the best separation between groups is obtained with JTpc interval. A selected value of JTpc $>203$ ms gave $76 \%$ in both sensitivity and specificity. In the ratios, QTp/QT ratio is significantly larger in OMI patients than in normal subjects, and the Tpe/QTp and Tpe/QT ratios are significantly larger in normal subjects

Table 1

Mean values of the durations of the ventricular activity intervals and HR in the study groups

\begin{tabular}{|c|c|c|c|c|c|c|c|}
\hline$m(\mathrm{sd})$ & QTc & QTpc & JTc & JTpc & Tpe & QRS & HR \\
\hline Normal & $414(23)$ & $290(20)$ & $310(16)$ & 187 (14) & $114(8)$ & 95 (10) & $71(8)$ \\
\hline Patients & $423(26)$ & 320 (19) & $323(24)$ & $220(17)$ & $96(13)$ & $94(10)$ & $69(8)$ \\
\hline$p$ & 0.27 & $6 \times 10^{-4}$ & 0.09 & $1.9 \times 10^{-5}$ & $1.7 \times 10^{-4}$ & 0.58 & 0.21 \\
\hline
\end{tabular}

Values are given as mean (sd), intervals in milliseconds, and HR in beats/min. $p$ values are from the Wilcoxon rank sum test.

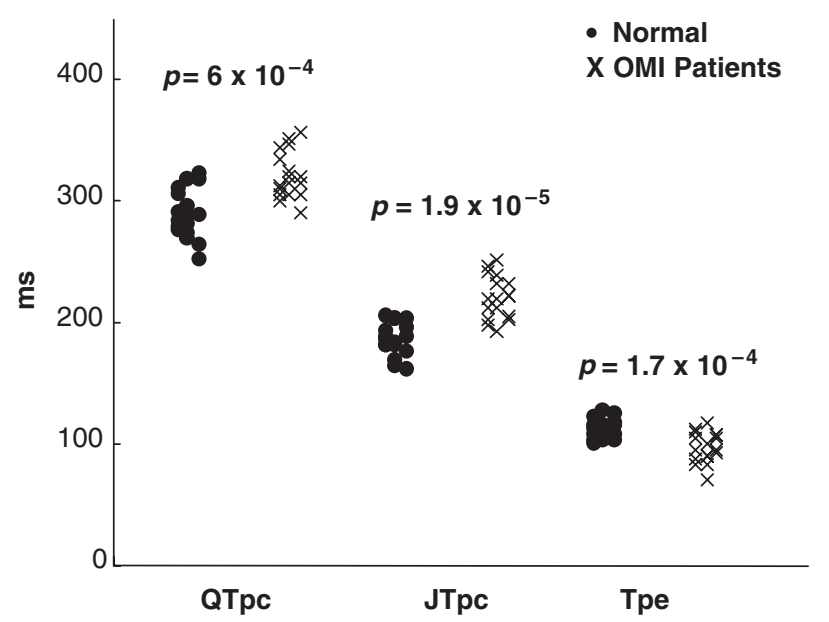

Fig. 1. QTpc, JTpc and Tpe intervals in the study groups ( $p$ values are from the Wilcoxon rank sum test). 
Table 2

Measures of intervals and ratios with $p$ and cutoff values, sensitivity and specificity in the study groups

\begin{tabular}{lllllll}
\hline & Normal & OMI patients & $p$ value & Cutoff value & Sensitivity (\%) & Specificity (\%) \\
\hline QTpc & $290(20)$ & $320(19)$ & $6 \times 10^{-4}$ & 305 & 70 & 70 \\
JTpc & $187(14)$ & $220(17)$ & $1.9 \times 10^{-5}$ & 203 & 76 & 76 \\
Tpe & $114(8)$ & $96(13)$ & $1.7 \times 10^{-4}$ & 105 & 70 & 76 \\
QTp/QT & $0.70(0.02)$ & $0.75(0.03)$ & $8.1 \times 10^{-7}$ & 0.72 & 94 & 76 \\
Tpe/QTp & $0.39(0.04)$ & $0.30(0.04)$ & $3.6 \times 10^{-6}$ & 0.35 & 94 & 82 \\
Tpe/QT & $0.28(0.02)$ & $0.23(0.02)$ & $1.4 \times 10^{-5}$ & 0.25 & 94 & 82 \\
JTp/JT & $0.60(0.02)$ & $0.68(0.03)$ & $6.7 \times 10^{-7}$ & 0.63 & 100 & 94 \\
Tpe/JTp & $0.66(0.07)$ & $0.47(0.07)$ & $6.3 \times 10^{-7}$ & 0.56 & 100 & 94 \\
Tpe/JT & $0.39(0.02)$ & $0.31(0.03)$ & $5.4 \times 10^{-7}$ & 0.36 & 100 & 94 \\
\hline
\end{tabular}

Values of intervals (in milliseconds) and ratios of normal and OMI patients are given as mean (sd).

Cutoff values of intervals are given in milliseconds.

$p$ values are from the Wilcoxon rank sum test.

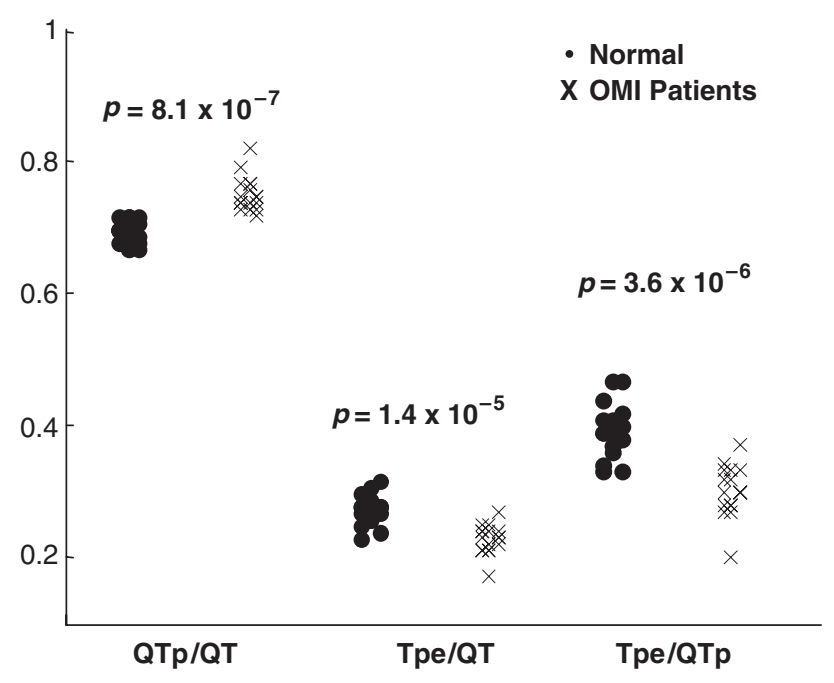

Fig. 2. QTp/QT, Tpe/QT and Tpe/QTp ratios in the study groups ( $p$ values are from the Wilcoxon rank sum test).

than in OMI patients (Fig. 2). The best separation between groups is obtained with the Tpe/QTp and Tpe/QT ratios. Selection of cutoff values of Tpe/QTp $<0.35$ and Tpe/QT $<0.25$ gave $94 \%$ sensitivity and $82 \%$ specificity in these ratios.

All the proposed ratios better discriminate between groups than the ventricular repolarization intervals and other ratios (Fig. 3). The JTp/JT ratio is significantly larger in OMI patients than in normal subjects $\left[(0.68 \pm 0.03)\right.$ vs. $\left.(0.60 \pm 0.02), p=6.7 \times 10^{-7}\right]$; and the Tpe/JTp and Tpe/JT ratios are significantly larger in normal subjects than OMI patients [ $(0.66 \pm 0.07)$ vs. $(0.47 \pm 0.07), p=6.3 \times 10^{-7}$, and $(0.39 \pm 0.02)$ vs. $(0.31 \pm 0.03), p=5.4 \times 10^{-7}$, respectively]. Selection of cutoff values of JTp/JT $>0.63, \mathrm{Tpe} / \mathrm{JTp}<0.56$ and Tpe $/ \mathrm{JT}<0.36$ gave $100 \%$ sensitivity and $94 \%$ specificity in these ratios. 


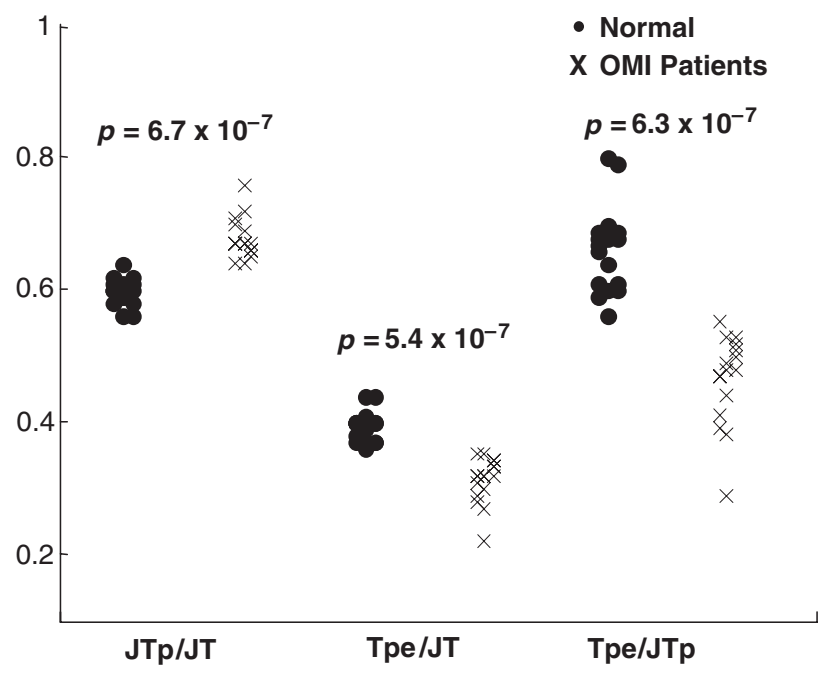

Fig. 3. JTp/JT, Tpe/JT and Tpe/JTp ratios in the study groups ( $p$ values are from the Wilcoxon rank sum test).

\section{Discussion and conclusions}

Our results show that the novel ratios proposed to evaluate the ventricular repolarization improve the separation between OMI patients and normal subjects, compared with the commonly used indices of duration of the ventricular repolarization intervals and other ratios. We observed that the QTp and JTp intervals prolong and the Tpe shortens in OMI patients when these intervals are measured in the infarct related leads.

In this study, some relevant factors influencing the duration of the QT interval, such as age, gender, HR and QRS duration, do not contribute any significant difference. For the measurement of time intervals, automated and robust computer algorithms have been developed using wavelet analysis to accurately detect the points of interest of the QRS complex and $T$ wave. These algorithms were validated using the CSE multi-lead measurement database [16], and the measurements were within the tolerance limits for deviations with respect to the measurements by experts [19,21].

Another important factor influencing the variability of QT interval measurements in the 12-lead ECG is that there is not any established criterion for lead selection [2,22]. In this study, the QT interval has been measured from the leads that correspond to the closest QTmax approximations: V3 for normal subjects [22] and, in post-MI patients, V2 or V6 for patients with anterior infarct, and D2 or aVF for patients with inferior infarct, that correspond to the site of the infarct [23].

In agreement with previous studies, the QTc, QTpc, JTc, and JTpc intervals in our patients are longer than in normal subjects $[4,6,8,24]$. The differences are significant only for the QTpc and JTpc intervals, and the best separation between groups is obtained with the JTpc interval. The Tpe interval is significantly shorter in the patients' group. This could be a consequence of the shortening of action potentials with ischemia $[13,25]$.

The ratios proposed to evaluate the ventricular repolarization: JTp/JT, Tpe/JTp and Tpe/JT are more precise in separating OMI patients from normal subjects than the duration of the ventricular repolarization intervals and other ratios. These ratios indicate that changes in the $T$-wave morphology reflect alterations 
of the repolarization due to the chronic infarct and ischemia. Because in a large proportion of patients with coronary artery disease the combination of ischemia and scar is probably responsible for the genesis of lethal arrhythmias [26], these ratios could improve the identification of OMI patients at high risk of ventricular tachyarrhythmias. Future work will focus on corroborating these findings in a larger population of OMI patients with and without susceptibility to ventricular tachyarrhythmias.

\section{Summary}

Prolonged QT interval has been reported as a risk factor of ventricular arrhythmias in post-MI patients, but its sensitivity and specificity is low. We tested the hypothesis that ratios between some ventricular repolarization intervals improve the separation between normal subjects and patients with OMI. These novel indices of ventricular repolarization are based on JT, JTp and Tpe intervals, the JTp/JT, Tpe/JTp and Tpe/JT ratios. These indices have been compared with the duration of the intervals: QT, QTp, JT, JTp and Tpe and ratios: QTp/QT, Tpe/QTp and Tpe/QT in 17 normal subjects and 17 OMI patients. The computer algorithms to detect the points of interest in the QRS complex and $T$ wave were based on the WT and were validated in 25 records of the CSE multi-lead measurement database. The intervals has been measured from V3 for normal subjects and, V2 or V6 for patients with anterior infarct, and D2 or aVF for patients with inferior infarct. In the ventricular repolarization intervals and intervals ratios, the best separation between groups is obtained with the Tpe/QTp and Tpe/QT ratios. These ratios are significantly larger in normal subjects than in OMI patients. Selection of cutoff values of Tpe/QTp $<0.35$ and Tpe/QT $<0.25$ gave $94 \%$ sensitivity and $82 \%$ specificity in these ratios. In the proposed ratios, JTp/JT ratio is significantly larger in OMI patients than in normal subjects $\left[(0.68 \pm 0.03)\right.$ vs. $\left.(0.60 \pm 0.02), p=6.7 \times 10^{-7}\right]$. The Tpe/JTp and Tpe/JT ratios are significantly larger in normal subjects than OMI patients $[(0.66 \pm 0.07)$ vs. $(0.47 \pm 0.07), p=6.3 \times 10^{-7}$, and $(0.39 \pm 0.02)$ vs. $(0.31 \pm 0.03), p=5.4 \times 10^{-7}$, respectively] Selection of cutoff values of JTp/JT $>0.63$, Tpe $/ \mathrm{JTp}<0.56$ and Tpe $/ \mathrm{JT}<0.36$ gave $100 \%$ sensitivity and $94 \%$ specificity in these ratios. Therefore, these ratios are more precise in separating OMI patients from normal subjects than the intervals and other ratios. These indices should be further tested to determine their usefulness in the identification of OMI patients with risk of ventricular arrhythmias.

\section{Acknowledgements}

This work has been funded by a scholarship from the CONACYT (México) to Carlos Alvarado-Serrano and by project SAF98-0120 from the Spanish CYCIT.

\section{References}

[1] B. Surawicz, S.B. Knoebel, Long QT: good bad or indifferent?, J. Am. Coll. Cardiol. 4 (1984) $398-413$.

[2] P. Davey, QT interval and mortality from coronary artery disease, Prog. Cardiovasc. Dis. 42 (2000) 359-384.

[3] W. Zareba, A.J. Moss, and the LQTS Study Group, Criteria for delayed repolarization in patients with wide QRS complex, J. Am. Coll. Cardiol. 23 (1994) 37A.

[4] L. Oikarinen, M. Viitasalo, L. Toivonen, Dispersions of the QT interval in postmyocardial infarction patients presenting with ventricular tachycardia or with ventricular fibrillation, Am. J. Cardiol. 81 (1998) 694-697. 
[5] W. Zareba, A.J. Moss, S. leCessie, Dispersion of ventricular repolarization and arrhythmic cardiac death in coronary artery disease, Am. J. Cardiol. 74 (1994) 550-553.

[6] L. Oikarinen, L. Toivonen, M. Viitasalo, Electrocardiographic measures of ventricular repolarization dispersion in patients with coronary artery disease susceptible to ventricular fibrillation, Heart 79 (1998) 554-559.

[7] P. Davey, QT interval measurement $Q$ : to $T$ apex or $Q$ to Tend?, J. Int. Med. 246 (1999) 145-149.

[8] I. Savelieva, Y.G. Yap, G. Yi, X. Guo, A.J. Camm, M. Malik, Comparative reproducibility of QT, QTpeak and TpeakTend intervals and dispersion in normal subjects, patients with myocardial infarction, and patients with hypertrophic cardiomyopathy, Pacing Clin. Electrophysiol. 21 (Pt. II) (1998) 2376-2381.

[9] J. Benhorin, M. Merri, M. Alberti, E. Locati, W.J. Hall, A.J. Moss, The long QT syndrome: new electrocardiographic characteristics, Circulation 82 (1990) 521-527.

[10] M. Zabel, S. Portnoy, M.R. Franz, Electrocardiographic indexes of dispersion of ventricular repolarization: an isolated heart validation study, J. Am. Coll. Cardiol. 25 (1995) 746-752.

[11] C. Antzelevitch, V.V. Nesterenko, W. Shimizu, J.M. Di Diego, Electrophysiological characteristics of the M-cell, in: M.R. Franz, C. Schmitt, B. Zrenner (Eds.), Monophasic Action Potentials, Springer, Berlin/Heidelberg, Germany, 1997.

[12] A. Lubinski, Z. Kornacewics-Jach, A.M. Wnuk-Wojnar, J. Adamus, M. Kempa, T. Królak, E. Lewicka-Nowak, M. Radomski, G. Swiatecka, The terminal portion of the $T$ wave: a new electrocardiographic marker of risk of ventricular arrhythmias, Pacing Clin. Electrophysiol. 23 (Pt. II) (2000) 1957-1959.

[13] J. O'Donnell, D.E. Lovelace, S.B. Knoebel, P.L. McHenry, Behavior of the terminal $T$ wave during exercise in normal subjects, patients with symptomatic coronary artery disease and apparently healthy subjects with abnormal ST segment depression, J. Am. Coll. Cardiol. 5 (1985) 78-84.

[14] I. Savelieva, Y.G. Yap, G. Yi, X. Guo, K. Hnatkova, A.J. Camm, M. Malik, Relation of ventricular repolarization to cardiac cycle length in normal subjects, hypertrophic cardiomyopathy, and patients with myocardial infarction, Clin. Cardiol. 22 (10) (1999) 649-654.

[15] M. Zabel, P.R. Lichtlen, A. Haverich, M.R. Franz, Comparison of ECG variables of dispersion of ventricular repolarization with direct myocardial repolarization measurements in the human heart, J. Cardiovasc. Electrophysiol. 9 (1998) $1279-1284$.

[16] J.L. Willems, P. Arnaud, J.H. Van Bemmel, R. Degani, P.W. Macfarlane, C. Zywietz for the CSE Working Party, Common standards for quantitative electrocardiography: goals and main results. Method. Inf. Med. 29 (1990) 263-271.

[17] J. Ramos, Detection of high-frequency atrial micropotentials, Ph.D. Thesis, Department of Electronic Engineering, Universitat Politècnica de Catalunya, Barcelona, Spain, 1997; available from: http://petrus.upc.es/ wwwdib/tesis/Jramos/ resumen.html (in Spanish).

[18] H.H. Friedman, Diagnóstico electrocardiográfico y vectocardiográfico, 3a. Ed., Salvat, Barcelona Spain, 1989 (in Spanish).

[19] C. Alvarado, Analysis of the variability of ECG time intervals, Ph.D. Thesis, Department of Electronic Engineering, Universitat Politècnica de Catalunya, Barcelona, Spain, 2001; available from: http://www.tdx.cesca.es/ TDX-1126102-154204 (in Spanish).

[20] J.L. Willems, Common standards for quantitative electrocardiography, CSE Multilead Atlas, Measurement Results-Data Set 3, CSE Project, ACCO Publication, Leuven Belgium, 1988.

[21] The CSE working party, Recommendations for measurement standards in quantitative electrocardiography, Eur. Heart J. 6 (1985) 815-825.

[22] J.C. Cowan, K. Yusoff, M. Moore, P.A. Amos, A.E. Gold, J.P. Bourke, S. Tansuphaswadikul, R.W.F. Campbell, Importance of lead selection in QT interval measurement, Am. J. Cardiol. 61 (1988) 83-87.

[23] M. Yamaki, H. Igarashi, K. Ikeda, I. Kubota, K. Tsuiki, S. Yasui, The body surface distribution of the QT interval in patients with previous myocardial infarction and normal subjects, Jpn. Circ. J. 51 (1987) 1289-1295.

[24] J.S. Perkiomaki, M.J. Koistinen, S. Yli-Mayry, H.V. Huikuri, Dispersion of QT interval in patients with and without susceptibility to ventricular tachyarrhythmias after previous myocardial infarction, J. Am. Coll. Cardiol. 26 (1995) 174-179.

[25] A.G. Kleber, M.J. Janse, F.J.L. van Capelle, D. Durrer, Mechanism and time course of $S$ - $T$ and $T$ - $Q$ segment changes during acute regional myocardial ischemia in the pig heart determined by extracellular and intracellular recordings, Circ. Res. 42 (1978) 603-613.

[26] D. Metha, J. Curwin, J.A. Gomes, V. Fuster, Sudden death in coronary artery disease: acute ischemia versus myocardial substrate, Circulation 96 (1997) 3215-3223. 
Carlos Alvarado-Serrano received the Electronics and Communications Engineer degree from National Polytechnic Institute (IPN), México D.F. in 1981, M.Sc. degree in Bioelectronics from Research Center and Advanced Studies of the IPN (CINVESTAV), México D.F. in 1992 and Eng. Dr. degree from Technical University of Catalonia (UPC), Barcelona, Spain in 2001. He is a Professor in the Bioelectronics Section of the Electrical Engineering Department at CINVESTAV and teaches courses in medical and electronic instrumentation. His research interests include biomedical signal processing, instrumentation methods and sensors.

Juan Ramos received the Telecommunication Engineering and Eng. D. degrees from the Universidad Politècnica de Catalunya (UPC), Barcelona, Spain, in 1992 and 1997, respectively. In 1992, he joined the Electronic Engineering Department, as a Lecturer and, since 1997, he has been an Associate Professor, teaching courses in several areas of electronic instrumentation. He is a member of the Biomedical Research Center at the UPC. His research interests include biomedical and electronic instrumentation.

Ramon Pallàs-Areny received the Ingeniero Industrial and Doctor Ingeniero Industrial degrees from the Technical University of Catalonia (UPC), Barcelona, Spain, in 1975 and 1982, respectively.

$\mathrm{He}$ is a Professor of Electronic Engineering at the same University, and teaches courses in medical and electronic instrumentation at the Castelldefels School of Technology. In 1989 and 1990 he was a visiting Fulbright Scholar and, in 1997 and 1998 he was an Honorary Fellow at the University of Wisconsin, Madison. In 2001 he was nominated Professor Honoris Causa by the Faculty of Electrical Engineering of the University of Cluj-Napoca (Romania). His research includes instrumentation methods and sensors based on electrical impedance measurements, sensor interfaces, noninvasive physiological measurements and electromagnetic compatibility in electronic systems. He is the author of six books and the leading author of other five books on instrumentation in Spanish and Catalan. He is also coauthor (with John G. Webster) of Sensors and Signal Conditioning, 2nd ed. (New York: Wiley, 2001), and Analog Signal Processing (New York: Wiley, 1999).

Dr. Pallàs-Areny was a recipient, with John G. Webster, of the 1991 Andrew R. Chi Prize Paper Award from the Instrumentation and Measurement Society (IEEE). In 2000 he received the Award for Quality in Teaching granted by the Board of Trustees of the UPC, and in 2002 the Narcís Monturiol Medal from the Autonomous Government of Catalonia. 